\title{
Transcriptome-wide analysis of differentially expressed chemokine receptors, SNPs, and SSRs in the age-related macular degeneration
}

Madhu Sudhana Saddala ${ }^{1,2+}$, Anton Lennikov ${ }^{1,2+}$ D , Anthony Mukwaya ${ }^{3}$, Lijuan Fan ${ }^{1,2}$, Zhengmao Hu ${ }^{4}$ and Hu Huang ${ }^{1,2,5^{*}}$

\begin{abstract}
Background: Age-related macular degeneration (AMD) is the most common, progressive, and polygenic cause of irreversible visual impairment in the world. The molecular pathogenesis of the primary events of AMD is poorly understood. We have investigated a transcriptome-wide analysis of differential gene expression, single-nucleotide polymorphisms (SNPs), indels, and simple sequence repeats (SSRs) in datasets of the human peripheral retina and RPE-choroid-sclera control and AMD.

Methods and results: Adaptors and unbiased components were removed and checked to ensure the quality of the data sets. Molecular function, biological process, cellular component, and pathway analyses were performed on differentially expressed genes. Analysis of the gene expression datasets identified 5011 upregulated genes, 11,800 downregulated genes, 42,016 SNPs, 1141 indels, and 6668 SRRs between healthy controls and AMD donor material. Enrichment categories for gene ontology included chemokine activity, cytokine activity, cytokine receptor binding, immune system process, and signal transduction respectively. A functional pathways analysis identified that chemokine receptors bind chemokines, complement cascade genes, and create cytokine signaling in immune system pathway genes ( $p$ value $<0.001$ ). Finally, allele-specific expression was found to be significant for Chemokine (C-C motif) ligand (CCL) 2, 3, 4, 13, 19, 21; C-C chemokine receptor (CCR) 1, 5; chemokine (C-X-C motif) ligand (CXCL) 9, 10, 16; C-X-C chemokine receptor type (CXCR) 6; as well as atypical chemokine receptor (ACKR) 3,4 and pro-platelet basic protein (PPBP).
\end{abstract}

Conclusions: Our results improve our overall understanding of the chemokine receptors' signaling pathway in AMD conditions, which may lead to potential new diagnostic and therapeutic targets.

Keywords: AMD, RNA-Seq, Gene ontology, Human eye, Chemokine receptor

\section{Background}

Age-related macular degeneration (AMD) is the most common, progressive, and polygenic disease responsible for visual impairment in individuals over the age of 60 [1]. The number of individuals with AMD has steadily increased, and it is estimated to reach 196 million people

\footnotetext{
* Correspondence: huangh1@missouri.edu

†Madhu Sudhana Saddala and Anton Lennikov have contributed equally to this work.

${ }^{1}$ Mason Eye Institute, University of Missouri, Columbia, MO 65212, USA

2Wilmer Eye Institute, Johns Hopkins University, Baltimore, MD 21287, USA

Full list of author information is available at the end of the article
}

affected by 2020, given the increasing longevity of the worldwide population [2]. Because AMD is a progressive degenerative disease, having the intermediate stage puts one at risk for the more advanced forms of AMD. Clinically, two forms of advanced AMD are recognized: geographic atrophy and neovascular AMD. Geographic atrophy is characterized by the loss of an area of the RPE and choroid that results in the gradual decline in the number of photoreceptors. Neovascular or "wet" form of AMD is characterized by the growth of abnormal new blood vessels from the choroid into sub-RPE

(C) The Author(s). 2019 Open Access This article is distributed under the terms of the Creative Commons Attribution 4.0 International License (http://creativecommons.org/licenses/by/4.0/), which permits unrestricted use, distribution, and 
and subretinal regions. These poorly developed neovessels cause hemorrhage and exudation of fluid in the macular region interfering with the function of the retina leading to loss of vision. Even though treatments aimed at inhibiting blood vessel growth can effectively slow the progression of "wet" AMD, no useful treatments exist for the atrophic ("dry") form of the disease, which accounts for the majority of all AMD cases [3]. Several biological processes have been implicated in the pathogenesis of AMD, including complement activation [4], inflammation [5], and oxidative stress [6]. Studies of gene expression regarding the AMD phenotype are becoming increasingly important in assessing the relevance and possible functions of AMD risk loci and in moving beyond genetic association to uncover the specific pathways involved in the development and progression of the disease [7, 8]. Many of the studies to date investigating AMD gene association are based on genome-wide association (GWA) [9] such as association with HtrA serine peptidase 1 (HTRA1)/age-related maculopathy susceptibility 2 (ARMS2) and CFH [10, 11]. However, most of the variants investigated so far are tag single-nucleotide polymorphisms (SNPs) or noncoding SNPs within large intergenic regions, thus making it difficult to ascertain the function of disease-associated variants. Coding variants can cause truncated transcripts that can affect protein synthesis, and function or stability. Here, RNA-Seq allows for a global analysis of the transcriptome of the affected tissue in an unbiased manner with the capacity to measure the expression of individual genes [6]. Moreover, RNA-Seq analysis can also assess the different isoforms of each gene, alternative splice events, novel, and rare transcripts, and identify the role of ncRNAs. RNA-Seq also has a lower frequency of falsepositive rates and a higher reproducibility $[12,13]$.

Understanding which genes are perturbed in the pathophysiology of AMD could pave the way for the identification of biomarkers as potential predictors of disease onset and can be validated as potential therapeutical targets. Also, the identification of potential therapies may be facilitated by high-throughput systems using biological analyses, particularly at the transcriptome levels.

Global gene expression assays, however, only provide information about the transcriptome profile at the time of sampling which in turn can be affected by the rate of translation, and by the rate of protein turnover [14], thus, all genomics, proteomics, and transcriptome analysis methodologies are required for a complete understanding of gene and protein interaction involved in the AMD pathophysiology.

Here, publicly available RNA-Seq datasets representing total mRNA harvested from human ocular tissues from healthy and AMD donors were analyzed using unique analysis pipelines. The dataset was analyzed for differentially expressed genes (DEGs) and using these DEGs, pathway enrichment analysis and functional annotation analyses were performed. From the results, we identify the most dysregulated pathways such as chemokine signaling pathway, complement cascade pathway, and cytokine signaling, and biological processes, such as cell communication, cell surface receptor signaling pathway, signal transduction, biological adhesion, and numerous chemokine receptors, which may be significant players in the pathophysiology of AMD.

\section{Methods}

\section{Data source, quality, and preprocessing}

The human healthy and AMD condition in the retina and RPE-choroid-scleral (RCS) RNA-Seq raw paired-end datasets obtained from the Sequence Read Archive (SRA) (https:/www.ncbi.nlm.nih.gov/sra/?term=SRP107937) from the National Centre for Biotechnology Information (NCBI). Kim et al. produced the original dataset. [15] Eight AMD and healthy Caucasian close age-matched donor eyes datasets were used, PMI $<6 \mathrm{~h}$. The mean age for AMD donors was $84.1 \pm 6.9$ years, and the healthy donor was $85 \pm 3.1$ years. Donors in AMD group were diagnosed histologically with early-stage non-exudative age-related maculopathy (three donors, six datasets); late stage nonexudative age-related maculopathy (four donors, eight datasets); and RPE cell epithelial dystrophy (one donor, two datasets). Donors in the healthy control group were selected based on the absence of ocular pathology. Equal weight was assigned to retinal and RPE/choroid material with a dataset from the same donor. Detailed information of datasets is presented in Table 1.

Kim et al. who created and deposited the datasets focused on the significant differential expression of noncoding RNA and antisense transcripts in AMD [15], whereas our current work focuses on chemokines receptors and their pathways, in the pathophysiology of AMD, using bioinformatics analysis pipeline (Fig. 1), using the SRA Toolkit (https://www. ncbi.nlm.nih.gov/sra/docs/toolkitsoft/). The SRA files were converted to a fastq format into forward and reverse separate files using the SRA Toolkit split function. These raw reads were used for visualization of the reads' quality before and after preprocessing by using FastQC software (https://www.bioinformatics.babraham.ac.uk/ projects/fastqc/). The process to remove adapters and ambiguous quality reads were done using the Trimmomatic0.36 tool, here trimming of bases from $3^{\prime}$ and $5^{\prime}$ ends, maintaining the Phred-score at $\leq 30$.

\section{Reference-based assembly}

The human genome was aquired from the NCBI genome (https://www.ncbi.nlm.nih.gov/genome/?term=human) for reference-based assembly. All the datasets were assembled separately with reference genomes using Bowtie software [16]. Initially, Bowtie indexed the genome with a 
Table 1 Detailed information of age macular degeneration (AMD) datasets with experiment, runs, sex, age, donor id, eye histological phenotype, and tissue [15]

\begin{tabular}{|c|c|c|c|c|c|c|}
\hline Experiment & Run & Sex & Age & Donor ld & Eye histological phenotype & Tissue \\
\hline SRX2848433 & SRR5591599 & Male & 85 & Normal_1 & Normal & Peripheral retina \\
\hline SRX2848434 & SRR5591600 & Male & 85 & Normal_1 & Normal & Peripheral RPE-choroid-sclera \\
\hline SRX2848435 & SRR5591601 & Male & 84 & Normal_2 & Normal & Peripheral retina \\
\hline SRX2848436 & SRR5591602 & Female & 92 & Normal_3 & Normal & Peripheral retina \\
\hline SRX2848437 & SRR5591603 & Female & 92 & Normal_3 & Normal & Peripheral RPE-choroid-sclera \\
\hline SRX2848438 & SRR5591604 & Female & 86 & Normal_4 & Normal & Peripheral retina \\
\hline SRX2848439 & SRR5591605 & Female & 86 & Normal_4 & Normal & Peripheral RPE-choroid-sclera \\
\hline SRX2848440 & SRR5591606 & Male & 83 & Normal_5 & Normal & Peripheral retina \\
\hline SRX2848441 & SRR5591607 & Male & 83 & Normal_5 & Normal & Peripheral RPE-choroid-sclera \\
\hline SRX2848442 & SRR5591608 & Male & 83 & Normal_6 & Normal & Peripheral retina \\
\hline SRX2848443 & SRR5591609 & Male & 83 & Normal_6 & Normal & Peripheral RPE-choroid-sclera \\
\hline SRX2848444 & SRR5591610 & Male & 84 & Normal_7 & Normal & Peripheral retina \\
\hline SRX2848445 & SRR5591611 & Male & 84 & Normal_7 & Normal & Peripheral RPE-choroid-sclera \\
\hline SRX2848446 & SRR5591612 & Female & 83 & Normal_8 & Normal & Peripheral retina \\
\hline SRX2848447 & SRR5591613 & Female & 83 & Normal_8 & Normal & Peripheral RPE-choroid-sclera \\
\hline SRX2848448 & SRR5591614 & Male & 69 & $\mathrm{AMD}_{-} 1$ & Early age-related maculopathy & Peripheral retina \\
\hline SRX2848449 & SRR5591615 & Male & 69 & $\mathrm{AMD}_{-} 1$ & Early age-related maculopathy & Peripheral RPE-choroid-sclera \\
\hline SRX2848450 & SRR5591616 & Female & 85 & AMD_2 & Early age-related maculopathy & Peripheral retina \\
\hline SRX2848451 & SRR5591617 & Female & 85 & AMD_2 & Early age-related maculopathy & Peripheral RPE-choroid-sclera \\
\hline SRX2848452 & SRR5591618 & Male & 95 & AMD_3 & Late non-exudative age-related maculopathy & Peripheral retina \\
\hline SRX2848453 & SRR5591619 & Male & 95 & AMD_3 & Late non-exudative age-related maculopathy & Peripheral RPE-choroid-sclera \\
\hline SRX2848454 & SRR5591620 & Male & 87 & $\mathrm{AMD}_{-} 4$ & Late exudative age-related maculopathy & Peripheral retina \\
\hline SRX2848455 & SRR5591621 & Male & 87 & AMD_4 & Late exudative age-related maculopathy & Peripheral RPE-choroid-sclera \\
\hline SRX2848456 & SRR5591622 & Female & 83 & AMD_5 & Late non-exudative age-related maculopathy & Peripheral retina \\
\hline SRX2848457 & SRR5591623 & Female & 83 & AMD_5 & Late non-exudative age-related maculopathy & Peripheral RPE-choroid-sclera \\
\hline SRX2848458 & SRR5591624 & Female & 86 & AMD_6 & Other (RPEcell epithelial dystrophy (suspected)) & Peripheral retina \\
\hline SRX2848459 & SRR5591625 & Female & 86 & AMD_6 & Other (RPEcell epithelial dystrophy (suspected)) & Peripheral RPE-choroid-sclera \\
\hline SRX2848460 & SRR5591626 & Female & 86 & $\mathrm{AMD}_{-} 7$ & Late non-exudative age-related maculopathy & Peripheral retina \\
\hline SRX2848461 & SRR5591627 & Female & 86 & AMD_7 & Late non-exudative age-related maculopathy & Peripheral RPE-choroid-sclera \\
\hline SRX2848462 & SRR5591628 & Female & 86 & AMD_8 & Early age-related maculopathy & Peripheral retina \\
\hline SRX2848463 & SRR5591629 & Female & 86 & AMD_8 & Early age-related maculopathy & Peripheral RPE-choroid-sclera \\
\hline
\end{tabular}

Burrows-Wheeler index to keep its memory footprint small. Finally, the RNA-Seq by expectation-maximization (RSEM) tool extracted the reference transcripts from a genome with gene annotations in a GTF file. All the annotation files were merged to obtain a single assembly file [17]. We obtained good assembly results with the human dataset when validating the assembly read remapping that was conducted using bowtie 2 for each data set, creating the bowtie 2 index [16]. CAP3 assembler was used to keep only the longest isoform for each gene to reduce redundancy [18].

\section{Identification of DEGs}

Trinity software supports the use of Bioconductor tool such as EdgeR to analyze differential expression analysis in the assembled transcriptome. Finally, the both healthy controls and AMD comparison transcript counts (matrix file) were used for differential gene expression using edgeR (Empirical analysis of digital gene expression in $\mathrm{R}$ ) package of Bioconductor with primary parameters such as false discovery rate (FDR), log fold-change (logFC), log counts per million $(\log C P M)$, and $p$ value $[19,20]$. Unigenes with adjusted $q$ value less than $0.001 \quad(p<0.001)$ and the fold changes more than $4(\log \mathrm{FC}>4)$ were considered as significantly differentially expressed unigenes.

\section{Functional annotation}

Gene ontology (GO) is an internationally standardized gene functional classification system that offers a dynamically 


\section{RNA-seq Processing Pipeline}

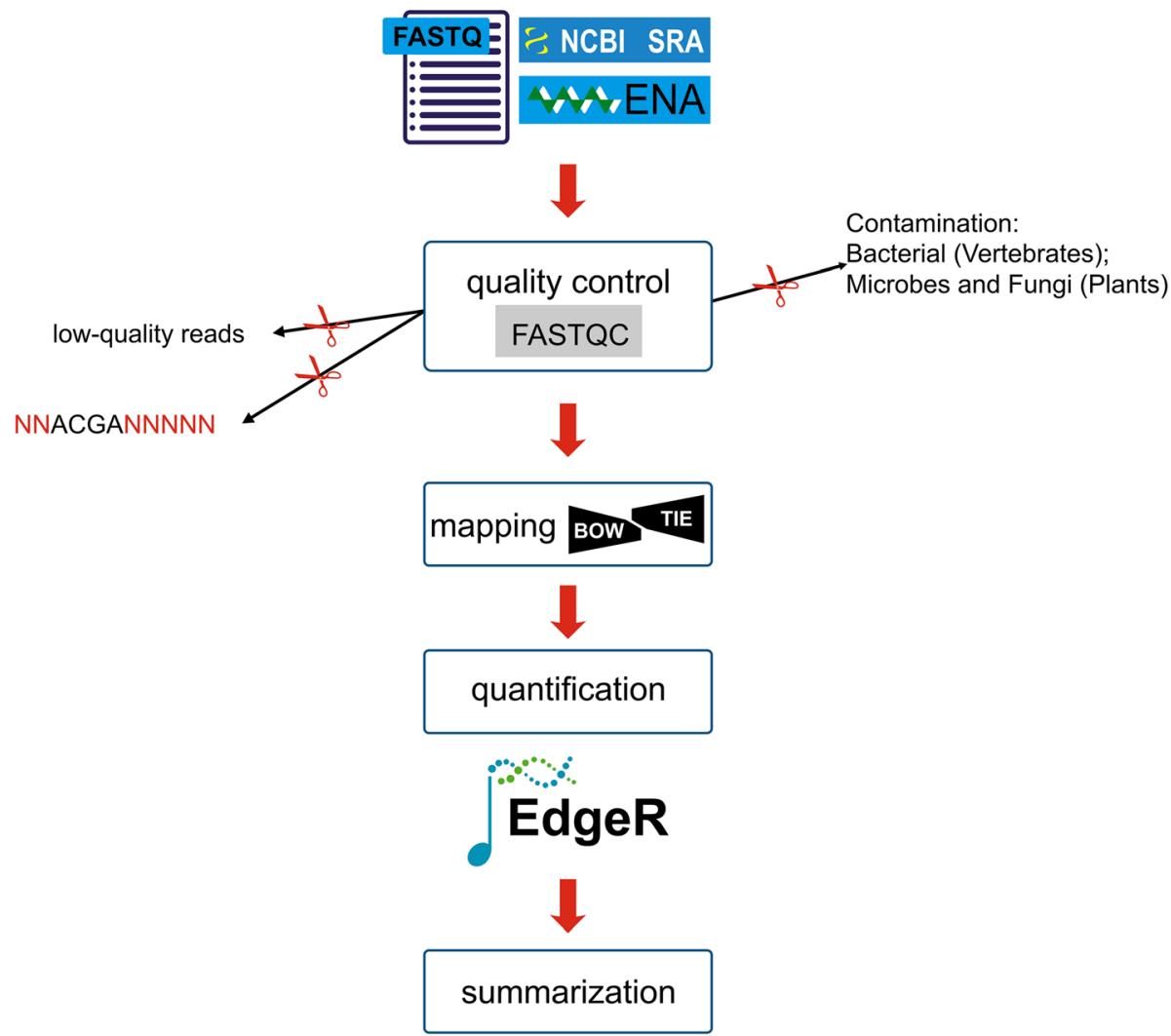

Fig. 1 The basic workflow of RNA-Seq analysis. The human data sets collected, quality checked, reference genome mapped, and gene expression quantification. Gene ontology, functional pathways, gene network analysis, and summarization

updated controlled vocabulary and a strictly defined concept to comprehensively describe the properties of genes and their products in any organism. The identified DEGs were subjected to further functional analysis using the GO enrichment analysis [21]. DEGs were assigned to their biologically relevant pathways using the KEGG pathway database through the KEGG Automated Annotation Server (KAAS) [22]. Hypergeometric test using the GO terms and the KEGG pathway (Kyoto Encyclopedia of Genes and Genomes, http://www.kegg.jp/) terms were performed, and the analysis of significant GO terms or KEGG pathway terms were identified with a $p$ value $<0.05$.

\section{Identification of SNPs and indels}

The good quality clean reads from each sample were aligned individually to reference the transcriptome using a Burrows-Wheeler Aligner (BWA). The read count profile from the output file (.sam file) of the BWA alignment was generated by using samtools. For SNP identification, the sorted alignment files from each sample were used to produce a bcf file using samtools [23]. The bcf tool was used to filter the SNPs at a $p$ value $<0.001$. The potential SNPs were identified using read depth $(d) \geq 10$, quality depth
$(Q) \geq 30$, minimum root mean square mapping quality $(\mathrm{MQ}) \geq 40$, and flanking sequence length $(l)=50$.

\section{Identification of simple sequence repeats}

The identification of simple sequence repeats (SSRs) in the unigenes of healthy and AMD human datasets was predicted using the Perl script MISA (MIcroSAtellite; http://pgrc.ipk-gatersleben.de/misa) tool [24]. Dinucleotide repeats of more than six times and tri, tetra, penta, and hexanucleotide repeats of more than five times were considered the search criteria for SSRs.

\section{Gene network analysis}

We also performed the functional enrichment and interaction network analysis using the STRING 10.5 database [25] on using the DEGs as the input files. STRING tool classified the DEGs according to the GO categories, such as biological processes (BP), molecular function (MF), cellular components (CC), and Kyoto Encyclopedia of Genes and Genomes database (KEGG) pathways. 


\section{Results}

\section{Quality and pre-processing of datasets}

The RNA-Seq paired-end human healthy and AMD data (SRP107937) were acquired from the National Centre for Biotechnology Information-Sequence Read Archive (NCBI-SRA) using the SRA Toolkit (https://www.ncbi. nlm.nih.gov/sra/docs/toolkitsoft/) with a prefetch function, save for one file (SRR5591614). The paired 30 SRA files were converted into fastq files (60 files) with fastq-dump and split-files functions.

Initially, visualization of the quality of all datasets before and after trimming the adaptors and going through the pre-processing steps was performed using a FastQC tool (https://www.bioinformatics.babraham. ac.uk/projects/fastqc/). Finally, low-quality reads were removed by trimming the bases from $3^{\prime}$ and $5^{\prime}$ end and maintaining the Phred-score $\leq 30$ using the Trimmomatic-0.36 tool [26]. After cleaning and trimming of low-quality reads and adaptor removal, more than $97 \%$ good quality reads in each stage were retained. The human healthy and AMD of paired-end raw data before and after elimination of adapters and retained reads percentages were tabulated (Additional file 1: Table S1). These cleaned reads were used for further transcriptome assembly analysis.

\section{Reference-based assembly}

The healthy and AMD datasets were assembled separately with a reference human genome using Bowtie software [16]. The RSEM (RNA-Seq by expectation-maximization) tool can extract reference transcripts from a genome with gene annotations in a GTF file. From this extraction, 93\% and $95 \%$ of the reads from healthy and AMD, respectively, were successfully mapped on a reference transcriptome. All the annotation files were merged to obtain a single assembly file [17]. The assembled files were used for removing redundant sequences with CAP3 assembler software [18]. However, the CAP3 assembler generated 139,575 contigs and 1,610,520 singlets. Both the contig and singlet files were combined, and sequences below 300 in length were removed. The final output file was considered the reference genome for further analysis. All the samples were given equal weight within a control dataset and a disease dataset. Therefore, all samples $(n=8)$ control (neural retina and RPE/choroid) vs. all AMD comparison (neural retina and RPE/choroid) were used for the analysis.

\section{Differential gene expression analysis}

RSEM software was used to calculate the expression values in the form of fragments per kilobase of exon per million mapped reads (FRKM). Finally, both the healthy and AMD comparison transcript counts (matrix file) were used for differential gene expression using edgeR (empirical analysis of digital gene expression in R) package of Bioconductor with primary parameters such as the false discovery rate (FDR), log fold change (logFC), $\log$ counts per million $(\log C \mathrm{PM})$, and $p$ value Unigenes with a $p$ value less than $0.001 \quad(p<0.001)$ and a fold change of more than $4(\log \mathrm{FC}>4)$ were considered significantly differentially expressed genes. Principle component analysis (PCA) was performed for all the samples in both groups, and the results demonstrated that samples within the AMD group were separate from those in the control group. Furthermore, PCA screen plot confirmed that principle component 1 (PC1) and 2 (PC2) accounted for $98 \%$ of the total variation in gene expression. To further investigate the two groups, we performed hierarchical clustering of all DEGs. Extract clusters of transcripts with similar expression profiles by cutting the transcript cluster dendrogram at a given percent of its height (60\%), creating individual transcript clusters and summarizes expression values for each cluster according to individual charts (Additional file 1: Figure S2). We discovered 5011 upregulated and 11,800 downregulated DEGs among the 51,532 totals, in AMD conditions relative to healthy controls datasets. The hierarchical clustering heatmap, correlation matrix plot, MA plot, and volcano plots were generated to represent the up- and downregulated genes (Fig. 2).

\section{Functional annotation}

In the current study, functional assignment and categorization for the identified DEGs were carried out using a GO analysis by the GO enrichment analysis [21]. All the up- and downregulated genes were uploaded to the GO enrichment analysis tool with the complete human genome as the background. The MFs, BPs, CC protein classes, and pathways were predicted in the significantly enriched GO terms.

The upregulated genes were involved in various molecular functions, such as protein binding (139 genes), receptor activity (77 genes), receptor binding (73 genes), $\mathrm{G}$ protein-coupled receptor activity (34 genes), cytokine activity (16 genes), cytokine receptor binding (10 genes), chemokine activity (10 genes), and extracellular matrix structural constituent (8 genes), respectively (Fig. 3a). The upregulated genes were involved in various biological processes, such as RNA metabolic process (33 genes), cell communication (150 genes), cell surface receptor signaling pathway (70 genes), signal transduction (142 genes), biological adhesion (25 genes), cellular component movement (40 genes), regulation of molecular function (38 genes), immune system process (62 genes), cellular calcium ion homeostasis (19 genes), and cytokine-mediated signaling pathway (12 genes) (Fig. 3b). The upregulated genes were involved in various cellular components, such as nucleus (42 genes), macromolecular complex (47 genes), 


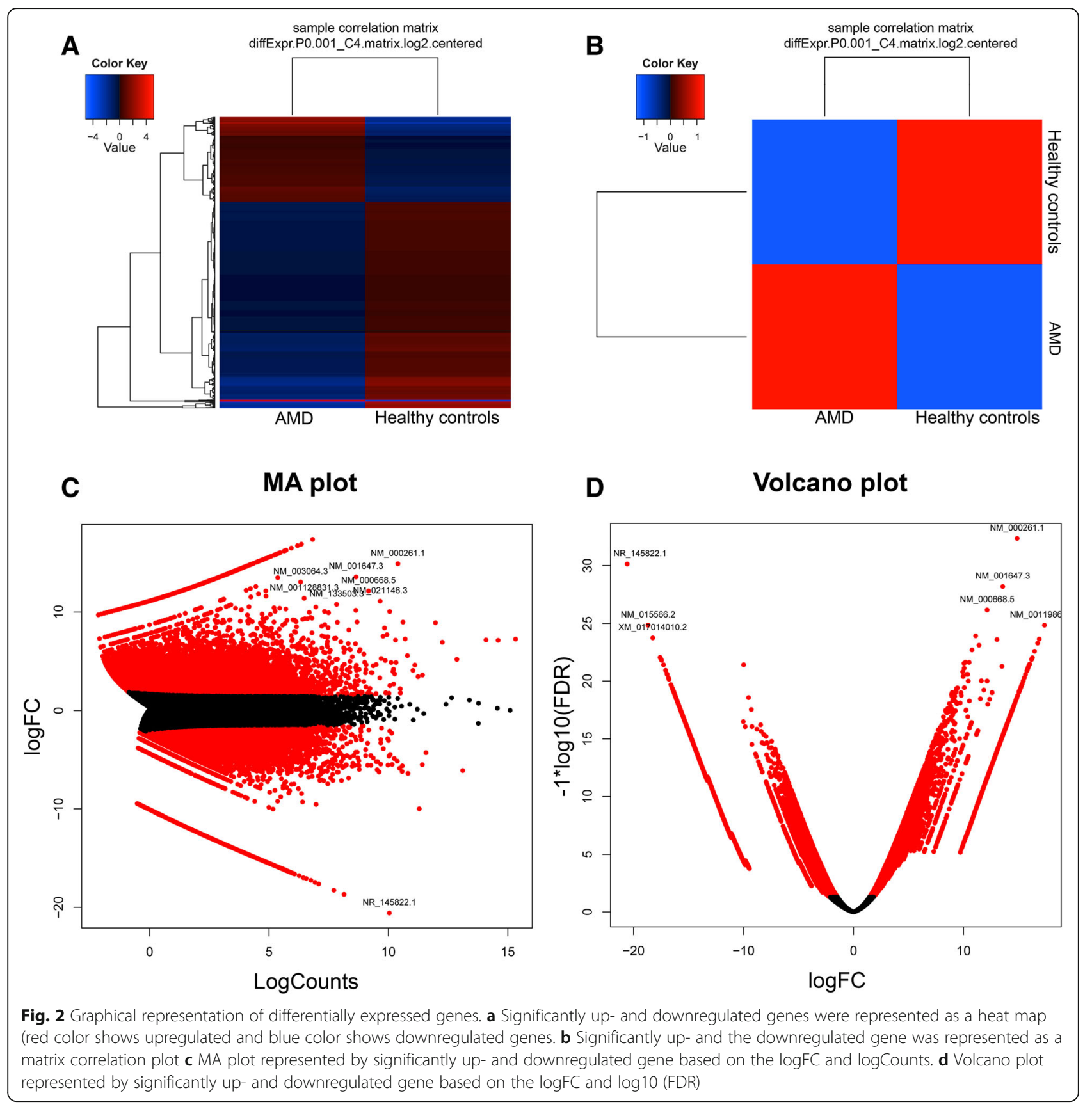

extracellular matrix (21 genes), protein complex (42 genes), organelle (101 genes), intracellular (150 genes), cell part (159 genes), extracellular space (59 genes), extracellular region (75 genes), and extracellular matrix (27 genes), respectively (Fig. 3c).

The downregulated genes were involved in various molecular functions, such as transporter activity (85 genes), transmembrane transporter activity (77 genes), ion channel activity (42 genes), ligand-gated ion channel activity (26 genes), and glutamate receptor activity (13 genes), respectively (Fig. 4a). The downregulated genes were involved in various biological processes, such as immune system process (10 genes), signal transduction (166 genes), cell surface receptor signaling pathway (92 genes), multicellular organismal process (136 genes), neurological system process (84 genes), nervous system development (36 genes), neurotransmitter secretion (13 genes), visual perception (13 genes), and neuron-neuron synaptic transmission (23 genes), respectively (Fig. 4b). The downregulated genes were involved in various cellular components, such as integral to membrane (79 genes), cell projection (46 genes), dendrite (15 genes), neuron projection (37 genes), synapse (21 genes), and postsynaptic membrane (9 genes), respectively (Fig. 4c). 


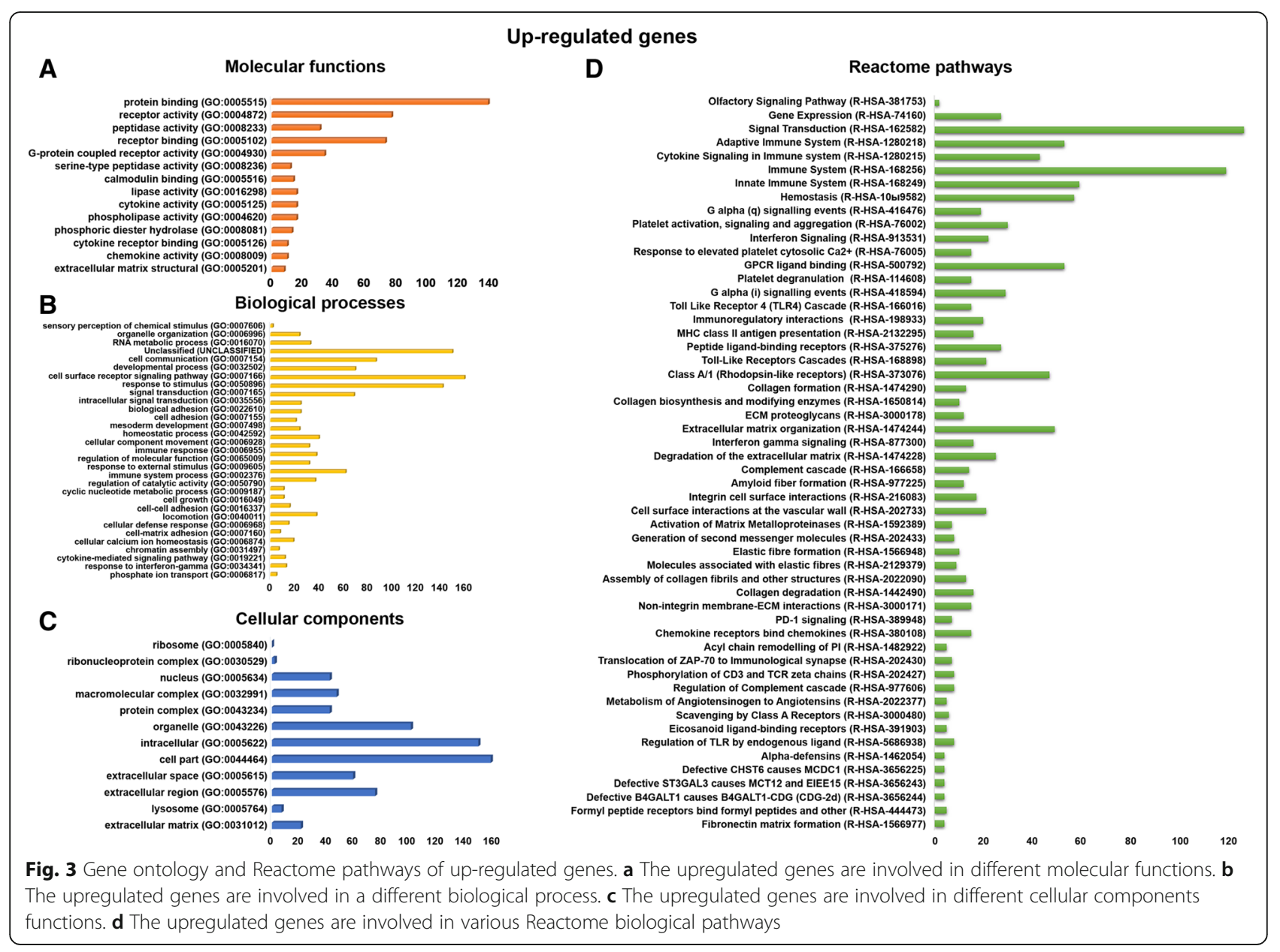

\section{Pathways analysis}

We identified the upregulated genes involved in different reactome pathways, such as extracellular matrix organization (49 genes), peptide ligand-binding receptors (27 genes), class A/1 (rhodopsin-like receptors) (47 genes), GPCR ligand binding (53 genes), signal transduction (126 genes), innate immune system (59 genes), immune system (119 genes), regulation of TLR by endogenous ligand (8 genes), toll-like receptors cascades (21 genes), metabolism of angiotensinogen to angiotensin ( 5 genes), complement cascade (14 genes), adaptive immune system (53 genes), chemokine receptors bind chemokines (15 genes), collagen degradation (16 genes), degradation of the extracellular matrix ( 25 genes), assembly of collagen fibrils and other multimeric structures (13 genes), collagen formation (13 genes), activation of matrix metalloproteinases (7 genes), cell surface interactions at the vascular wall (21 genes), hemostasis (57 genes), integrin cell surface interactions (17 genes), amyloid fiber formation (12 genes), interferon gamma signaling (16 genes), cytokine signaling in immune system pathway (43 genes), ECM proteoglycans (12 genes), MHC class II antigen presentation (16 genes), immunoregulatory interactions between a lymphoid and a non-lymphoid cell (20 genes), toll-like receptor 4 (TLR4) cascade (15 genes), G alpha (i) signaling events (29 genes), platelet activation, signaling, and aggregation (30 genes), G alpha (q) signaling events (19 genes), and unclassified pathways (388 genes) (Fig. 3d).

The downregulated genes were also involved in various Reactome pathways, such as phototransduction cascade (15 genes), visual phototransduction (21 genes), inactivation, recovery and regulation of the phototransduction cascade (14 genes), in neurotransmitter receptor binding and downstream transmission in the postsynaptic cell (34 genes), transmission across chemical synapses (46 genes), neuronal system (65 genes), GABA receptor activation (14 genes), activation of $\mathrm{G}$ protein-gated potassium channels (10 genes), inwardly rectifying $\mathrm{K}^{+}$channels (11 genes), potassium channels (21 genes), trafficking of AMPA receptors (8 genes), glutamate binding, activation of AMPA receptors and synaptic plasticity (8 genes), activation of kainate receptors upon glutamate binding (8 genes), and generic transcription pathway (72 genes), respectively (Fig. $4 \mathrm{~d}$ ).

In the current study, we primarily focused on chemokine receptors binding in the chemokines pathway, complements to the cascade pathway, and cytokine signaling in 


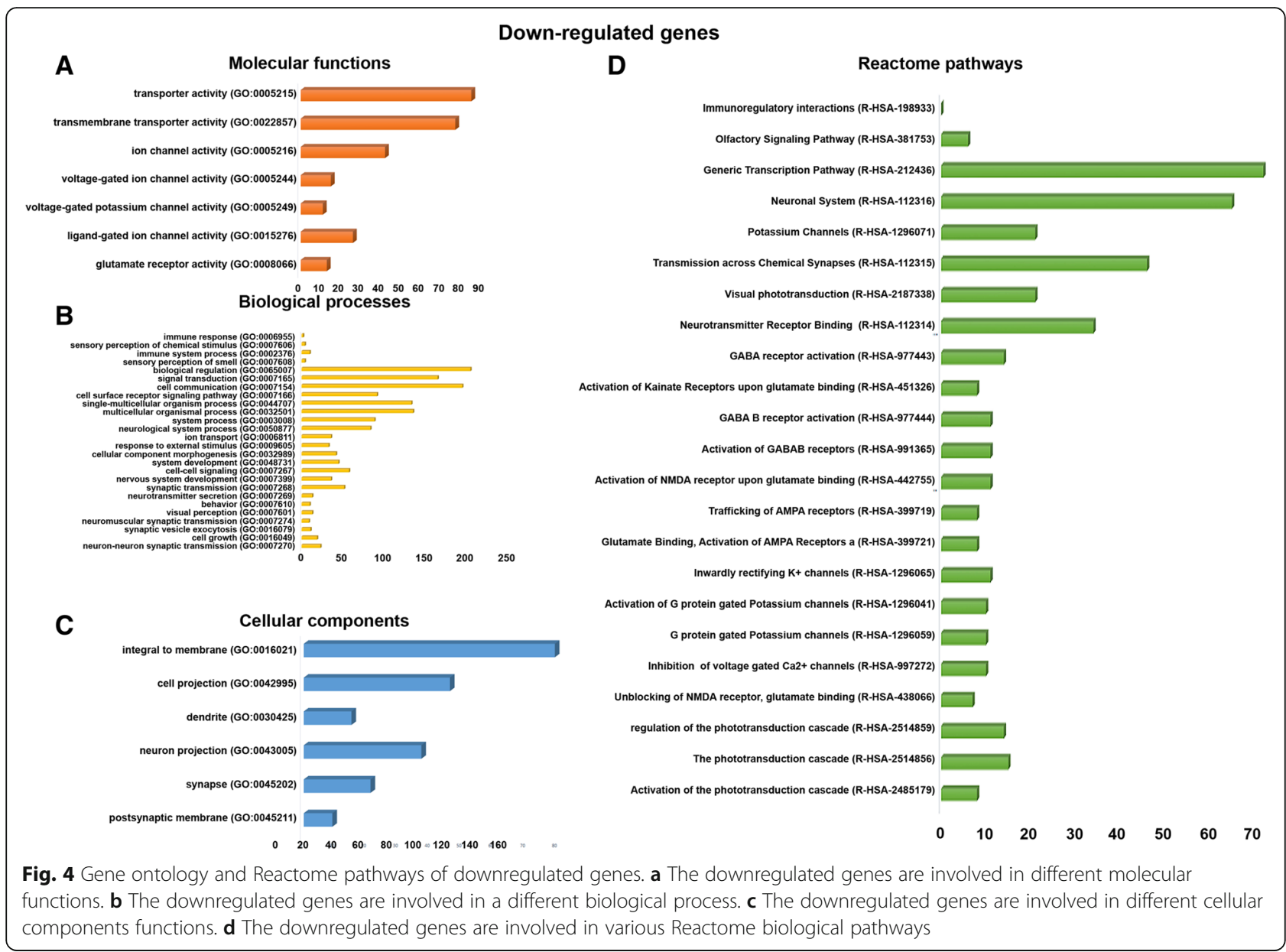

immune system pathways. CCL3, ACKR4, CCL19, CCL2, CXCL10, PPBP, CXCL9, CCL13, CCR1, CCL21, ACKR3, CXCL16, CCR5, CCL4, and CXCR6 are involved in the chemokine signaling pathway (Table 2). This pathway activates the JAK/Stat, Ras, ERK, and Akt pathways; NF-kappa B signaling pathway; and toll-like receptor signaling pathways. C5AR1, CFH, C1R, C5AR2, CFD, C1QB, C1QC, CD59, CFP, C3AR1, C3, $C R 1, C 1 Q A$, and $C 1 S$ are involved in the complement cascade pathway (Additional file 1: Table S2). There are 43 genes involved in cytokine signaling in the immune system pathways (Additional file 1: Table S3).

\section{SNPs and indels identification}

Since a significant number of SNPs and indels were initially mined, to narrow down the count, a quality value of 20 and read depth of 4 were applied as filtering criteria to identify significant SNPs and indels (Fig. 5a, b). We identified both the SNPs and indels in both the healthy and AMD human datasets; here, 114,513 SNPs were found in the healthy datasets, 42,016 SNPs were found in AMD datasets, and 145 SNPs were found in both the healthy and AMD datasets. For the indels, 4626 were found in healthy datasets, 1141 in AMD human datasets, and 14 in both data sets.

\section{SSRs markers identification}

We performed SSRs identification in human healthy and AMD datasets using the MISA tool. The results showed the total number of identified SSRs (6668), number of SSR containing sequences (3340), number of sequences containing more than one SSR (1546), and number of SSRs present in compound formation (618). Among all the identified SSRs, A/T single-nucleotide SSRs (4653) have a higher distribution in unigenes. Figure $5 \mathrm{c}$ represents the information of mononucleotides (4732), dinucleotides (985), trinucleotides (817), tetranucleotides (98), pentanucleotides (31), and hexanucleotides (5) repeats.

\section{Gene network analysis}

The up- and downregulated genes were used to construct gene-gene interactions using the STRING tool (https://string-db.org/), which also hid the disconnected nodes in the network. The results showed the analyzed number of nodes (581), expected number of edges (1113), a number of edges (1628), average node degree (5.6), 
Table 2 List of chemokine receptors bind chemokines pathway genes, UniProtKB, gene symbol, GenBank id, Gene name, logFC, $p$ value KEGG pathways

\begin{tabular}{|c|c|c|c|c|c|c|}
\hline UniProtKB & Gene symbol & GenBank ID & Gene name & $\log \mathrm{FC}$ & $p$ value & KEGG \\
\hline P10147 & CCL3 & NM_002983 & C-C motif chemokine 3 & 4.25663907 & 7.14E-07 & hsa:6349 \\
\hline Q9NPB9 & ACKR4 & NM_016557 & Atypical chemokine receptor 4 & 7.260759683 & $1.45 \mathrm{E}-14$ & hsa:51554 \\
\hline Q99731 & CCL19 & NM_006274 & C-C motif chemokine 19 & 12.30108718 & $2.93 \mathrm{E}-14$ & hsa:6363 \\
\hline P13500 & CCL2 & NM_002982 & C-C motif chemokine 2 & 5.52247071 & $6.79 \mathrm{E}-12$ & hsa:56477 \\
\hline P02778 & CXCL10 & NM_001565 & C-X-C motif chemokine 10 & 6.770426736 & 4.33E-09 & hsa:3627 \\
\hline P02775 & PPBP & NM_002704 & Platelet basic protein & 6.083239276 & 4.56E-11 & hsa:10895 \\
\hline Q07325 & CXCL9 & NM_002416 & C-X-C motif chemokine 9 & 7.708086768 & $2.59 \mathrm{E}-10$ & hsa:4283 \\
\hline Q99616 & CCL13 & NM_005408 & C-C motif chemokine 13 & 12.37011547 & $1.81 \mathrm{E}-14$ & hsa:6357 \\
\hline P32246 & CCR1 & NM_001295 & C-C chemokine receptor type 1 & 6.996085978 & 4.47E-16 & hsa:2826 \\
\hline 000585 & CCL21 & NM_002989 & C-C motif chemokine 21 & 7.794499691 & $5.22 \mathrm{E}-08$ & hsa:6366 \\
\hline P25106 & ACKR3 & NM_020311 & Atypical chemokine receptor 3 & 5.141169632 & $6.05 \mathrm{E}-11$ & hsa:57007 \\
\hline Q9H2A7 & CXCL16 & NM_001100812 & C-X-C motif chemokine 16 & 5.167455173 & $1.45 \mathrm{E}-10$ & hsa:58191 \\
\hline P51681 & CCR5 & NM_001100168 & C-C chemokine receptor type 5 & 5.636608022 & 7.70E-09 & hsa:1234 \\
\hline P13236 & CCL4 & NM_002984 & C-C motif chemokine 4 & 7.794499691 & $5.22 \mathrm{E}-08$ & hsa:6351 \\
\hline 000574 & CXCR6 & XM_005264809 & C-X-C chemokine receptor type 6 & 4.131178122 & $3.85 \mathrm{E}-06$ & hsa:10663 \\
\hline
\end{tabular}

A Single Nucleotide Polymorphism sites

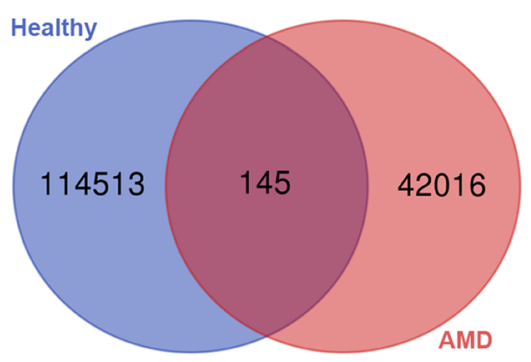

C

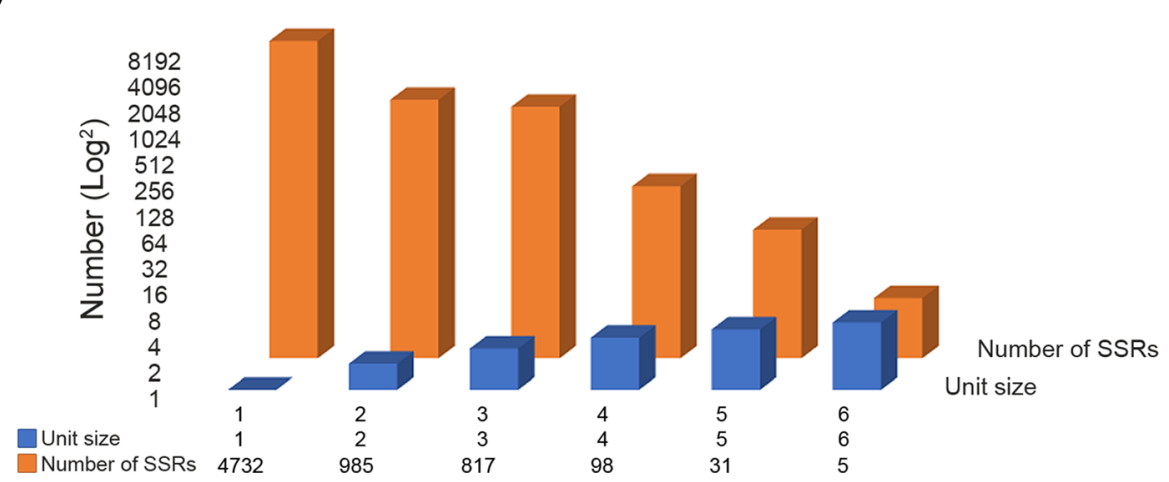

Fig. 5 Comparisons of control vs. human AMD of the differentially expressed genes of SNPs and Indels were illustrated by Venn diagrams. a The Venn diagram is represented SNPs of differential expressed genes in control vs. human AMD. $\mathbf{b}$ The Venn diagram is represented Indels of differential expressed genes in control vs. human AMD. c The simple sequence repeats (SSRs) of differential expressed genes represented by bar diagram in human AMD 
average local clustering coefficient (0.374), and PPI enrichment $p$ value $<1.0 \mathrm{e}-16$. Overview of the nodes and total interactions tree is presented in Additional file 1: Figure S1.

Individual pathways nodes were isolated for the presentation of interactions. Figure 6 demonstrates that the chemokine signaling pathway (k04062) (Fig. 6a) has 15 nodes, 82 edges, 10.9 average node degree, 0.844 avg. local clustering coefficient, and 4 expected number of edges with a PPI enrichment $p$ value $<1.0 \mathrm{e}-16$. The complement pathway (k04610) (Fig. 6b) has 14 nodes, 44 edges, 6.29 average node degree, 0.695 avg. local clustering coefficient, and 1 expected number of edges with a PPI enrichment $p$ value $<1.0 \mathrm{e}-16$. The cytokine-cytokine receptor interactions pathway (k04060) (Fig. 6c) has 43 nodes, 164 edges, 7.63 average node degree, 0.69 avg. local clustering coefficient, and 14 expected number of edges with a PPI enrichment $p$ value $<1.0 \mathrm{e}-16$.

\section{Discussion}

The present study elucidates the complete transcriptome sequencing (RNA-Seq) data analysis of healthy controls and AMD donor human eye tissues. Functional classification and pathway analysis revealed that the chemokine signaling pathway, complement cascade pathway, and cytokine signaling in immune system pathway genes and several other pathways are upregulated in ocular tissues. Chemokine receptors are small protein-coupled receptor (GPCR) superfamily and are activated on binding their cognate ligands of the chemokine family. Chemokine receptor binding initiates a cascade of intracellular events which are dissociation of the receptor-associated heterotrimeric $G$ proteins into $\alpha$ and $\beta \gamma$ subunits. $G \alpha$ activates an enzyme known as phospholipase C (PLC) that is associated with the cell membrane. PLC cleaves phosphatidylinositol $(4,5)$-bisphosphate (PIP2) to form two

\section{A Chemokine receptors system}

\section{B Compliment system}
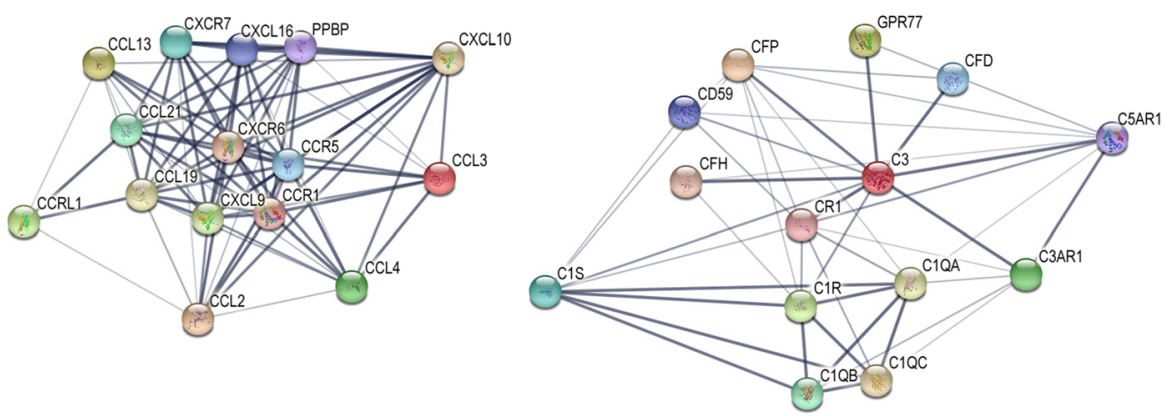

C

Cytokine system

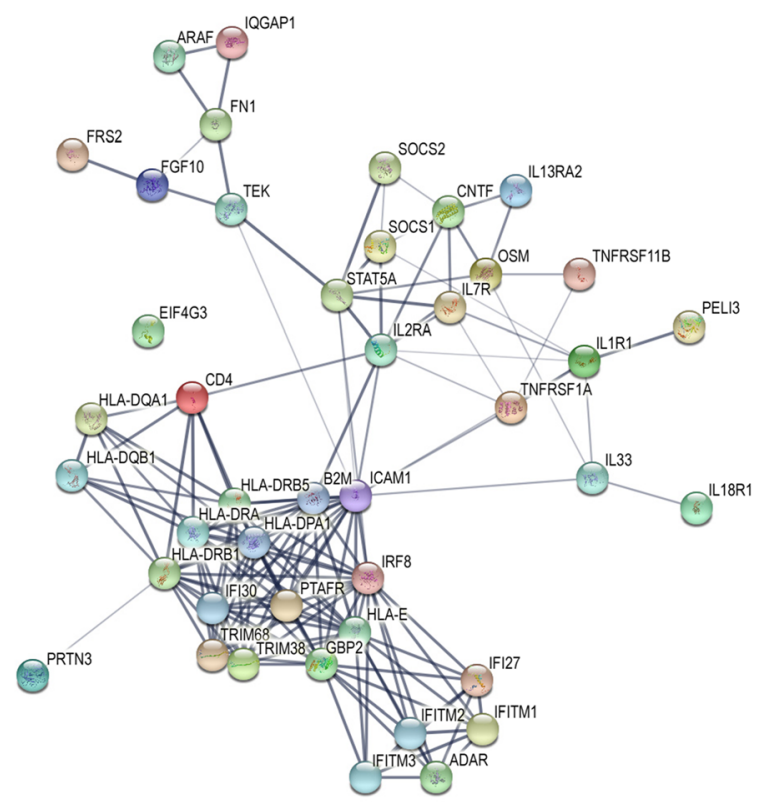

Fig. 6 The functional enrichment and gene network analysis. a Subnetwork of chemokine signaling pathway genes. b Subnetwork complement and coagulation cascades pathway genes. c Subnetwork cytokine-cytokine receptor interactions pathway genes 
second messenger molecules called inositol triphosphate (IP3) and diacylglycerol (DAG); DAG activates another enzyme called protein kinase C (PKC), and IP3 triggers the release of calcium from intracellular stores. These events promote many signaling cascades, affecting a cellular response. As per our results suggested that all the above-mentioned cascade signaling $G$ protein pathway genes, phospholipase $\mathrm{C}$ gene, protein kinase $\mathrm{C}$ gene, ion channel genes: GNA15, GNAO1, GNG3, GNG4, GNGT1, GNGT2, GRK4, GRK7, GPSM2, RGS3, PLCB2, PIK3CG, PTK2, PRKCZ, CACNA1A, CACNA1B, CACNA1E, and $K C N J 9$ are significantly upregulated in AMD conditions when compared with healthy controls.

Chemokines are also known to be upregulated in response to an infection and inflammation signals; they play critical roles in inflammatory and infectious diseases by regulating neutrophil, macrophage, and lymphocyte trafficking to the pathological sites [27, 28]. Leukocyte migration during conditions of tissue injury or infection is a multi-step process involving local upregulation of proinflammatory chemokine secretion in response to signaling molecules such as TNF- $\alpha$ and IFN- $\gamma$. Presentation of these chemokines on endothelial cell-surface glycosaminoglycans (GAGs), binding of chemokines to their cognate receptors on the leukocyte cell surface, activation of the leukocyte receptor, and migration of the leukocytes along the chemokine gradient within the extracellular matrix toward the site of chemokine release [29]. While in the eye, these processes are more complex due to the interplay of retinal microglia, RPE cells, and blood-retinal barrier (BRB) similar recruitment and activation of immune cells such as microglia is occurring.

Several chemokines implicated in AMD condition where CCL3 is reported as a critical regulator of retinal inflammation, which is associated with the severity of retinal degeneration and CCL3 is produced by subretinal microglia from the inner retina that can be a trigger for additional monocyte infiltration from the circulation via inner retinal blood vessels [30]. CCL19 is known to be involved in immune-regulatory and inflammatory processes [31]. CXCL7 is a protein belonging to the CXC-chemokine family and isoform of beta-thrombo-globulin or proplatelet basic protein (PPBP) [32]. Atypical chemokine receptor 4 (ACKR4) controls chemokine levels and localization via high-affinity chemokine binding and is uncoupled from classic ligand-driven signal transduction cascades, resulting in chemokine sequestration, degradation, or transcytosis [33]. CCL2 is involved in the neuroinflammatory processes that take place in the various diseases of the central nervous system (CNS), which are characterized by neuronal degeneration [34]. Nawaz et al. reported that autocrineautocrine CCL2, CXCL4, CXCL9, and CXCL10 signals in retinal endothelial cells are enhanced in diabetic retinopathy [35]. CCL13 is induced by the inflammatory cytokine interleukin-1 and TNF- $\alpha$ [36]. CXC-chemokine ligand 10 (CXCL10), which is a potent anti-angiogenic chemokine, is highly expressed in the retina of patients with AMD and laser-injured mice. Choroidal endothelial cells express CXC-chemokine receptor 3 (CXCR3), the CXCL10 receptor, and genetic ablation of CXCR3 exacerbates laser-induced choroidal neovascularization in mice [37]. Previous publications have indicated that some intraocular cytokine associated with inflammation decrease after anti-VEGF therapy in patients with AMD [38], and our results are consistent with these studies.

AMD is also associated with complementary activation or deregulation of the spontaneously initiated alternative complement pathway, leading to the local release of inflammatory activation products and local inflammation [39]. Multiple complement components, regulators, complement activation products, and inflammatory proteins have been identified to be upregulated in AMD conditions, including $C 3, C 3 d$; the terminal components $C 5$, $C 6, C 7, C 8$, and $C 9$; terminal complement regulators vitronectin and clusterin, apolipoproteins apoA1, apoA4, and apoE; as well as thrombospondin, serum amyloid $\mathrm{A}$ $(S A P-A)$, and SAP-P $[23,40]$. CFB is paralogous with complement component $2(C 2)$. These genes are in tandem in the major histocompatibility complex class III region, a cluster of immune-related genes on chromosome $6 \mathrm{p}$ and that carry AMD risk [41]. CFI is a co-factor along with $C F H$ for the inactivation of $C 3 b$. Noncoding polymorphisms adjacent to the gene and in an intron of CFI are associated with an altered risk of AMD [42]. The complement cascade is a soluble part of the innate immune system. Three different complement pathways activate enzyme cascades that ultimately lead to cell death. Each of the three complement pathways (alternative, lectin, and classical) is a unique method of activating the $\mathrm{C} 3$ molecule, initiating pro-inflammatory reactions, and activating the terminal complement pathway. Key to the amplification of the pathway is the fact that $\mathrm{C} 3$ is proteolytically activated to $\mathrm{C} 3 \mathrm{~b}$ due to $\mathrm{C} 3$ cleavage and forms a new C3 convertase molecule that will cleave more $\mathrm{C} 3$. This result in the production of $\mathrm{C} 3 \mathrm{bBb} 3 \mathrm{~b}$ in all pathways in addition to C4b2b3b in the classical and lectin pathways, molecules that act as $\mathrm{C} 5$ convertases [43]. The convertase cleaves C5 into C5a (an anaphylatoxin) and $\mathrm{C} 5 \mathrm{~b}$, which forms part of the membrane attack complex. C5b binds to $\mathrm{C} 6, \mathrm{C} 7, \mathrm{C} 8$, and $\mathrm{C} 9$ in sequence and $\mathrm{C} 8$ binds the incomplete membrane attack complex to cell surfaces and up to 16 C9 molecules are then assembled on the membrane to generate a circular polymer. The membrane attack complex can also bind to self-membranes, stimulating the release of growth factors from vascular endothelium. C3a, C4a, and C5a anaphylatoxins are released during these reactions. They are chemoattractants for phagocytic cells [44]. 
Furthermore, genetic variations (DNA polymorphisms) are known to be associated with phenotypic variation and may alter gene expression patterns [45]. A genetic locus at chromosome 10q26 provides strong susceptibility to AMD; specifically, two AMD-associated polymorphisms near ARMS2 and HTRA1 genes have been suggested to alter the gene expression of either one or the other gene [10]. One study recently reported that haplotypes with del443ins54 and rs11200638 variants influence HTRA1 and ARMS2 expression in genotyped human placentas [46]. However, another report presented that the del443ins54 variant does not change ARMS2 mRNA stability in lymphocytes [11], which is consistent with our data from retina samples. The polymorphic indel at the ARMS2 3' UTR was associated with AMD in Caucasian and Japanese data sets [47]. This indel is strongly associated with AMD.

There are a number of limitations of current work associated with the available dataset and focus of the current study on chemokine and complement pathways. First, the current analysis includes both retinal and RPE/ choroid material datasets that may lead to underrepresentation of retina-specific and RPE/choroid-specific DEG's output.

Second, only peripheral retinal material was available for the analysis as a limitation of original SRP107937 datasets generation; thus macular transcriptome events were potentially missing from the analysis. Third, human donor data early and late-stage AMD, as well as RPE degeneration samples were included in the analysis with the equal weight, thus potentially increasing heterogenicity. Fourth, retina and RPE/choroid datasets from the same donor were included as individual samples with equal weight. Further analytical efforts that can address some of the above-listed limitations and answer the question regarding tissue-specific events in retina and $\mathrm{RPE} /$ choroid are required.

\section{Conclusions}

The transcriptome-wide data analysis provides new insights into the differences in gene regulation between healthy and AMD conditions in the human eye. Analyses of gene ontology, functional pathway analysis, and transcriptional regulation networks of differentially expressed genes showed that chemokine activity, cytokine activity, and immune system activity are dominant during the AMD development, which is driven by the higher expression of the chemokine signaling pathway, complement cascade pathway, and cytokine signaling pathway genes. Among the DEGs, the CCL3, ACKR4, CCL19, CCL2, CXCL10, PPBP, CXCL9, CCL13, CCR1, CCL21, ACKR3, CXCL16, CCR5, CCL4, and CXCR6 (chemokine signaling pathway genes); C5AR1, CFH, C1R, C5AR2, CFD, C1QB, C1QC, CD59, CFP, C3AR1,
C3, CR1, C1QA, and C1S (complement cascade pathway genes); and cytokine signaling pathway genes were identified as potential novel regulators of AMD.

\section{Additional file}

Additional file 1: Figure S1. Gene network analysis. Chemokine signaling pathway (red color spheres), complement and coagulation cascades pathway (green color spheres) and cytokine-cytokine receptor interactions pathway (blue color spheres). Figure S2. A. Extract transcript clusters by expression profile by cutting the dendrogram. B. Principle component analysis, $X$ and $Y$ axis show principle component 1 and principle component 2 that explain $98 \%$ and $2 \%$ of the variance. Table S1. Human normal and AMD of paired-end raw data before and after removal of adapters and retained reads percentages. Table S2. List of complement cascade pathway genes, UniProtKB, gene symbol, GenBank id, Gene name, logFC, $p$-value KEGG pathways. Table S3. List of Cytokine signaling in immune system pathway genes, UniProtKB, gene symbol, GenBank id, Gene name, logFC, p-value KEGG pathways. (DOCX 920 kb)

\section{Acknowledgments}

The authors wish to acknowledge the contribution of the Center for Biomedical Informatics (CBMI) University of Missouri (Columbia, MO, USA) for computational resources. The RNA-Seq analysis workflow artwork was designed by Mr. Dmitry Rumyancev.

\section{Funding}

Hu Huang group is supported by NIH grant (EY027824), and University of Missouri startup funds.

\section{Availability of data and materials}

The datasets analyzed during the current study are available in the Sequence Read Archive repository, [https://www.ncbi.nlm.nih.gov/sra/?term=SRP107937].

\section{Authors' contributions}

The study was conceived and designed by MSS, AL, and HH. MSS have performed quality check, reference genome assembly, gene expression analysis, gene ontology, functional pathway analysis, and gene network analysis. LF and ZH have performed independent analysis validation. AL has conducted figures design and statistical analysis. The manuscript was written by MSS, AL, AM, LF, ZH, and $\mathrm{HH}$ and critically revised by $L F, Z H$, and $H H$. All authors reviewed and accepted the final version of the manuscript.

Ethics approval and consent to participate

In this study, only publicly available, anonymized data released under the Fort Lauderdale Agreement was analyzed, and thus, separate ethical approval was not required.

\section{Competing interests}

The authors declare that they have no competing interests.

\section{Publisher's Note}

Springer Nature remains neutral with regard to jurisdictional claims in published maps and institutional affiliations.

\section{Author details}

${ }^{1}$ Mason Eye Institute, University of Missouri, Columbia, MO 65212, USA. ${ }^{2}$ Wilmer Eye Institute, Johns Hopkins University, Baltimore, MD 21287, USA. ${ }^{3}$ Department of Ophthalmology, Faculty of Health Sciences, Institute for Clinical and Experimental Medicine, Linköping University, SE-581 83 Linköping, Sweden. ${ }^{4}$ Center for Medical Genetics and Hunan Key Laboratory of Medical Genetics, School of Life Sciences, Central South University, Changsha, China. ${ }^{5}$ Department of Ophthalmology, School of Medicine, University of Missouri-Columbia, 1 Hospital Drive, MA102C, Columbia, MO 65212, USA. 
Received: 4 January 2019 Accepted: 8 March 2019

Published online: 20 March 2019

\section{References}

1. Jager RD, Mieler WF, Miller JW. Age-related macular degeneration. N Engl J Med. 2008;358(24):2606-17

2. Wong WL, Su X, Li X, Cheung CM, Klein R, Cheng CY, Wong TY. Global prevalence of age-related macular degeneration and disease burden projection for 2020 and 2040: a systematic review and meta-analysis. Lancet Glob Health. 2014;2(2):e106-16.

3. Ambati J, Fowler BJ. Mechanisms of age-related macular degeneration. Neuron. 2012;75(1):26-39.

4. Anderson DH, Radeke MJ, Gallo NB, Chapin EA, Johnson PT, Curletti CR, Hancox LS, Hu J, Ebright JN, Malek G, et al. The pivotal role of the complement system in aging and age-related macular degeneration: hypothesis re-visited. Prog Retin Eye Res. 2010;29(2):95-112.

5. Wang Y, Wang VM, Chan CC. The role of anti-inflammatory agents in agerelated macular degeneration (AMD) treatment. Eye (Lond). 2011;25(2):127-39.

6. Khandhadia S, Lotery A. Oxidation and age-related macular degeneration: insights from molecular biology. Expert Rev Mol Med. 2010;12:e34.

7. McKay GJ, Patterson CC, Chakravarthy U, Dasari S, Klaver CC, Vingerling JR, Ho L, de Jong PT, Fletcher AE, Young IS, et al. Evidence of association of APOE with age-related macular degeneration: a pooled analysis of 15 studies. Hum Mutat. 2011;32(12):1407-16.

8. Reynolds R, Rosner B, Seddon JM. Serum lipid biomarkers and hepatic lipase gene associations with age-related macular degeneration. Ophthalmology. 2010;117(10):1989-95.

9. Fritsche LG, Igl W, Bailey JN, Grassmann F, Sengupta S, Bragg-Gresham JL, Burdon KP, Hebbring SJ, Wen C, Gorski M, et al. A large genome-wide association study of age-related macular degeneration highlights contributions of rare and common variants. Nat Genet. 2016;48(2):134-43.

10. Yang Z, Tong Z, Chen Y, Zeng J, Lu F, Sun X, Zhao C, Wang K, Davey L, Chen $\mathrm{H}$, et al. Genetic and functional dissection of HTRA1 and LOC387715 in age-related macular degeneration. PLoS Genet. 2010;6(2):e1000836.

11. Wang G, Spencer KL, Scott WK, Whitehead P, Court BL, Ayala-Haedo J, Mayo P, Schwartz SG, Kovach JL, Gallins P, et al. Analysis of the indel at the ARMS2 3'UTR in age-related macular degeneration. Hum Genet. 2010;127(5): 595-602.

12. Twine NA, Janitz K, Wilkins MR, Janitz M. Whole transcriptome sequencing reveals gene expression and splicing differences in brain regions affected by Alzheimer's disease. PLoS One. 2011;6(1):e16266.

13. Potok ME, Nix DA, Parnell TJ, Cairns BR. Reprogramming the maternal zebrafish genome after fertilization to match the paternal methylation pattern. Cell. 2013;153(4):759-72.

14. Weirick T, Militello G, Muller R, John D, Dimmeler S, Uchida S. The identification and characterization of novel transcripts from RNA-seq data. Brief Bioinform. 2016;17(4):678-85.

15. Kim EJ, Grant GR, Bowman AS, Haider N, Gudiseva HV, Chavali VRM Complete transcriptome profiling of normal and age-related macular degeneration eye tissues reveals dysregulation of anti-sense transcription. Sci Rep. 2018;8(1):3040

16. Langmead B, Salzberg SL. Fast gapped-read alignment with bowtie 2. Nat Methods. 2012;9(4):357-9.

17. Li B, Dewey CN. RSEM: accurate transcript quantification from RNA-Seq data with or without a reference genome. BMC Bioinformatics. 2011;12:323.

18. Huang X, Madan A. CAP3: a DNA sequence assembly program. Genome Res. 1999;9(9):868-77.

19. Robinson MD, McCarthy DJ, Smyth GK. edgeR: a Bioconductor package for differential expression analysis of digital gene expression data. Bioinformatics. 2010;26(1):139-40.

20. Anders S, Huber W. Differential expression analysis for sequence count data Genome Biol. 2010;11(10):R106

21. Mi H, Muruganujan A, Casagrande JT, Thomas PD. Large-scale gene function analysis with the PANTHER classification system. Nat Protoc. 2013, 8(8):1551-66.

22. Altschul SF, Lipman DJ. Protein database searches for multiple alignments. Proc Natl Acad Sci U S A. 1990:87(14):5509-13.

23. Li CM, Clark ME, Chimento MF, Curcio CA. Apolipoprotein localization in isolated drusen and retinal apolipoprotein gene expression. Invest Ophthalmol Vis Sci. 2006;47(7):3119-28.
24. Thiel T, Michalek W, Varshney RK, Graner A. Exploiting EST databases for the development and characterization of gene-derived SSR-markers in barley (Hordeum vulgare L.). Theor Appl Genet. 2003;106(3):411-22.

25. Szklarczyk D, Franceschini A, Kuhn M, Simonovic $M$, Roth $A$, Minguez $P$, Doerks T, Stark M, Muller J, Bork P, et al. The STRING database in 2011: functional interaction networks of proteins, globally integrated and scored. Nucleic Acids Res. 2011;39(Database):D561-8.

26. Bolger AM, Lohse M, Usadel B. Trimmomatic: a flexible trimmer for Illumina sequence data. Bioinformatics. 2014;30(15):2114-20.

27. Zlotnik A, Yoshie O. The chemokine superfamily revisited. Immunity. 2012; 36(5):705-16.

28. Bachelerie F, Graham GJ, Locati M, Mantovani A, Murphy PM, Nibbs R, Rot A Sozzani S, Thelen M. New nomenclature for atypical chemokine receptors. Nat Immunol. 2014;15(3):207-8.

29. Ebert LM, Schaerli $P$, Moser B. Chemokine-mediated control of T cell traffic in lymphoid and peripheral tissues. Mol Immunol. 2005;42(7):799-809.

30. Marques CP, Cheeran MC, Palmquist JM, Hu S, Urban SL, Lokensgard JR. Prolonged microglial cell activation and lymphocyte infiltration following experimental herpes encephalitis. J Immunol. 2008;181(9):6417-26.

31. Yoshida R, Imai T, Hieshima K, Kusuda J, Baba M, Kitaura M, Nishimura M, Kakizaki M, Nomiyama H, Yoshie O. Molecular cloning of a novel human CC chemokine EBI1-ligand chemokine that is a specific functional ligand for EBI1, CCR7. J Biol Chem. 1997:272(21):13803-9.

32. Hristov M, Zernecke A, Bidzhekov K, Liehn EA, Shagdarsuren E, Ludwig A, Weber C. Importance of CXC chemokine receptor 2 in the homing of human peripheral blood endothelial progenitor cells to sites of arterial injury. Circ Res. 2007;100(4):590-7.

33. Vinet J, van Zwam M, Dijkstra IM, Brouwer $N$, van Weering HR, Watts $A$, Meijer M, Fokkens MR, Kannan V, Verzijl D, et al. Inhibition of CXCR3mediated chemotaxis by the human chemokine receptor-like protein CCXCKR. Br J Pharmacol. 2013;168(6):1375-87.

34. Gerard C, Rollins BJ. Chemokines and disease. Nat Immunol. 2001:2(2):108-15.

35. Nawaz MI, Van Raemdonck K, Mohammad G, Kangave D, Van Damme J, Abu El-Asrar AM, Struyf S. Autocrine CCL2, CXCL4, CXCL9 and CXCL10 signal in retinal endothelial cells and are enhanced in diabetic retinopathy. Exp Eye Res. 2013;109:67-76.

36. Lamkhioued B, Garcia-Zepeda EA, Abi-Younes S, Nakamura H, Jedrzkiewicz S, Wagner L, Renzi PM, Allakhverdi Z, Lilly C, Hamid Q, et al. Monocyte chemoattractant protein (MCP)-4 expression in the airways of patients with asthma. Induction in epithelial cells and mononuclear cells by proinflammatory cytokines. Am J Respir Crit Care Med. 2000;162(2 Pt 1):723-32.

37. Fujimura S, Takahashi H, Yuda K, Ueta T, Iriyama A, Inoue T, Kaburaki T, Tamaki Y, Matsushima K, Yanagi Y. Angiostatic effect of CXCR3 expressed on choroidal neovascularization. Invest Ophthalmol Vis Sci. 2012:53(4):1999-2006

38. Sakamoto S, Takahashi $H$, Tan $X$, Inoue $Y$, Nomura $Y$, Arai $Y$, Fujino $Y$, Kawashima $H$, Yanagi $Y$. Changes in multiple cytokine concentrations in the aqueous humour of neovascular age-related macular degeneration after 2 months of ranibizumab therapy. Br J Ophthalmol. 2018;102(4): 448-54.

39. Donoso LA, Kim D, Frost A, Callahan A, Hageman G. The role of inflammation in the pathogenesis of age-related macular degeneration. Surv Ophthalmol. 2006:51(2):137-52.

40. Crabb JW, Miyagi M, Gu X, Shadrach K, West KA, Sakaguchi H, Kamei M, Hasan A, Yan L, Rayborn ME, et al. Drusen proteome analysis: an approach to the etiology of age-related macular degeneration. Proc Natl Acad Sci U S A. 2002;99(23):14682-7.

41. Gold B, Merriam JE, Zernant J, Hancox LS, Taiber AJ, Gehrs K, Cramer K, Neel J, Bergeron J, Barile GR, et al. Variation in factor B (BF) and complement component 2 (C2) genes is associated with age-related macular degeneration. Nat Genet. 2006;38(4):458-62.

42. Fagerness JA, Maller JB, Neale BM, Reynolds RC, Daly MJ, Seddon JM. Variation near complement factor I is associated with risk of advanced AMD. Eur J Hum Genet. 2009:17(1):100-4.

43. Yates JR, Sepp T, Matharu BK, Khan JC, Thurlby DA, Shahid H, Clayton DG, Hayward C, Morgan J, Wright AF, et al. Complement C3 variant and the risk of age-related macular degeneration. N Engl J Med. 2007;357(6):553-61.

44. Ricklin D, Hajishengallis G, Yang K, Lambris JD. Complement: a key system for immune surveillance and homeostasis. Nat Immunol. 2010;11(9):785-97.

45. Pastinen T, Ge B, Hudson TJ. Influence of human genome polymorphism on gene expression. Hum Mol Genet. 2006;15 Spec No 1:R9-16. 
46. Yu W, Dong S, Zhao C, Wang H, Dai F, Yang J. Cumulative association between age-related macular degeneration and less studied genetic variants in PLEKHA1/ARMS2/HTRA1: a meta and gene-cluster analysis. Mol Biol Rep. 2013:40(10):5551-61.

47. Gotoh N, Nakanishi H, Hayashi H, Yamada R, Otani A, Tsujikawa A, Yamashiro K, Tamura H, Saito M, Saito K, et al. ARMS2 (LOC387715) variants in Japanese patients with exudative age-related macular degeneration and polypoidal choroidal vasculopathy. Am J Ophthalmol. 2009;147(6):1037-41. 1041 e1031-1032

Ready to submit your research? Choose BMC and benefit from:

- fast, convenient online submission

- thorough peer review by experienced researchers in your field

- rapid publication on acceptance

- support for research data, including large and complex data types

- gold Open Access which fosters wider collaboration and increased citations

- maximum visibility for your research: over $100 \mathrm{M}$ website views per year

At $\mathrm{BMC}$, research is always in progress.

Learn more biomedcentral.com/submissions 